\title{
MORTANDAD CATASTRÓFICA DE GUANACOS POR ESTRÉS INVERNAL Y SUS IMPLICACIONES ARQUEOLÓGICAS: EL SITIO ALERO LOS GUANACOS 1, LAGO CARDIEL (PROVINCIA DE SANTA CRUZ, ARGENTINA)
}

\author{
DIEGO RINDEL*Y JUAN BAUTISTA BELARDI**
}

\begin{abstract}
RESUMEN
El objetivo de este trabajo es discutir las implicaciones tafonómicas y arqueológicas de episodios de mortalidad catastrófica de guanacos provocado por estrés invernal. Para ello se presentan los datos relevados en un sitio ubicado en la margen oeste del lago Cardiel. El conjunto faunístico fue caracterizado en función de las siguientes variables: estado de conservación, estructura etaria, posición de muerte, grado de enterramiento, grado de articulación, la acción de carnívoros, pisoteo y la asociación entre individuos y artefactos líticos. Se plantea que este tipo de eventos se presentan bajo determinadas condiciones que son normales en los ecosistemas del sur patagónico; asimismo, presentan una localización espacial predecible y redundante, fundamentalmente en lugares reparados. En términos tafonómicos, esto implica un potencial de mezcla de acumulaciones naturales y culturales en lugares utilizados como reparo tanto por el hombre como por los guanacos. Al considerar el impacto de este tipo de mortalidad de animales en las poblaciones humanas, se sugiere que, en el corto plazo, genera una oferta importante de recursos explotable a través de una estrategia de carroñeo. Esta es una opción poco costosa y de bajo riesgo (Borrero et al. 2005) que emplea una oferta inmediata que se presenta en una escala regional. Por estas razones, la implementación del carroñeo por parte de las poblaciones cazadoras recolectoras no sólo sería esperable en momentos iniciales de poblamiento, sino que pudo ser sistemáticamente utilizado a lo largo del tiempo. Por otra parte, debido a la baja diversidad que caracteriza a los ecosistemas del sur patagónico, la productividad de una región puede variar enormemente en el largo plazo. De esta manera, las fluctuaciones en la biomasa animal generadas por este tipo de mortalidad puede ser un factor que regule el abandono de determinados espacios, fundamentalmente aquellos asociados con una alta variabilidad climática como los cercanos a la cordillera.
\end{abstract}

PALABRAS CLAVE: estrés invernal, guanacos, tafonomía, carroñeo, cazadores recolectores.

* CONICET. Instituto Nacional de Antropología y Pensamiento Latinoamericano. 3 de Febrero 1370 (1426) Ciudad Autónoma de Buenos Aires, Argentina. drindelarqueo@yahoo.com

** Universidad Nacional de la Patagonia Austral. Unidad Académica Río Gallegos, Centro de Investigación Dra. Elsa Mabel Barbería. CONICET. Lisandro de la Torre 1070 (9400) Río Gallegos, Santa Cruz. Argentina. silespi@ infovia.com.ar 


\title{
CATASTROPHIC MORTALITY OF GUANACOS DUE TO WINTER STRESS AND ITS ARCHAEOLOGICAL IMPLICATIONS: ALERO LOS GUANACOS 1 SITE, LAGO CARDIEL
} (SANTA CRUZ, ARGENTINA)

\begin{abstract}
The aim of this paper is to present a case study that consists of an episode of catastrophic mortality of guanacos caused by winter stress in the west margin of Lago Cardiel (Santa Cruz, Argentina). The faunal assemblage is described in terms of the following variables: preservation state, age structure, death postures, degree of interment, disarticulation, carnivore marks, trampling, and association between carcasses and lithic artifacts. The taphonomic and archaeological implications of this kind of mortality events in guanacos are discussed. It is suggested that this kind of events occur under certain conditions which are normal in southern Patagonian ecosystems. Also, they show a predictable and redundant spatial location, mainly in sheltered places. In taphonomic terms, this implies a potential mixing of natural and cultural assemblages in places used as shelter by guanacos and humans. In terms of the impact of this kind of animal mortality events on human populations, it is suggested that, in the short term, they constitute an important supply of resources that is exploitable through a scavenging strategy. This is a low cost and low risk option (Borrero et al. 2005) which makes use of an immediate supply of the resource in question on a regional scale. Therefore, the implementation of a scavenging strategy by hunter-gatherers populations could be expected not only during the early peopling of the region, but also as a common strategy practiced through time. On the other hand, due to the low diversity that is characteristic of southern Patagonian environments, catastrophic death events can severely affect the productivity of a region in the long term. This implies, in turn, that the consequent fluctuations on animal biomass could constitute a factor controlling the abandonment of certain spaces, mainly those suffering a high climatic variability, such as areas near the Andean Cordillera.
\end{abstract}

KEY WORDS: winter stress, guanacos, taphonomy, scavenging, hunter gatherers.

\section{INTRODUCCIÓN}

Numerosos factores en la naturaleza son capaces de producir grandes acumulaciones óseas; entre ellos se cuentan la actividad de carnívoros (Binford 1981, Brain 1981, Martín y Borrero 1997, Mondini 1994, Pickering 2002, de Ruiter y Berger 2001, entre otros), accidentes (Lubinski y O 'B rien 2001), procesos atricionales (Behrensmeyer 1983, Behrensmeyer y DechantBoaz 1980, Borrero 2001), sequía (Conybeare y Haynes 1984, Haynes 1988, 1995, Shipman 1975), ahogamiento (Capaldo y Peters 1995), arrastre hidráulico (Behrensmeyer 1975, Gutierrez y Kaufmann 2004, Voorhies 1969) y humanos modernos (Binford 1978, O ' Connell et al. 1988, Yellen 1977a y b). A la vez, el estudio de conjuntos óseos generados por eventos de mortalidad masiva, entre los que se encuentran los provocados por estrés invernal, tiene una gran tradición dentro de la literatura tafonómica desde los comienzos de esta disciplina (Weigelt 1989). Distintos trabajos examinan la mortalidad por estrés invernal en varias especies de mamíferos grandes, tales como antílope pronghorn (Antilocapra americana) (Martinka 1967), caballos salvajes (Equus caballus) (Berger 1983) y antílope del Tíbet (Pantholops hodgsoni) (Schaller y J unrang 1988), entre otros.

En este trabajo se presenta un episodio de mortalidad catastrófica de guanacos (Lama guanicoe) provocado por estrés invernal en el año 2000 en el sitio Alero Los Guanacos 1, ubicado en la margen oeste del lago Cardiel (centro oeste de la provincia de Santa Cruz). En primer lugar, se brindan datos acerca de este proceso en ovinos y camélidos en la Patagonia, así como de los hábitos y estructura poblacional del guanaco, relevantes para la discusión del impacto de la mortalidad por estrés invernal en estas poblaciones. Luego se describe el sitio Alero Los Guanacos 1 y se caracteriza el conjunto faunístico en función de las siguientes variables: estado de preservación, estructura etaria, posición de muerte, grado de enterramiento, grado de articulación, la acción de carnívoros, pisoteo y la asociación en- 
tre individuos y artefactos líticos. Finalmente, se discuten distintas implicaciones de este tipo de mortalidad en guanacos para la arqueología del sur de Patagonia. Los ejes de la discusión giran en torno a la impronta diferencial de este tipo de eventos con respecto a otros procesos y agentes capaces de generar acumulaciones de estos animales, la mezcla potencial con restos arqueológi$\cos$, las posibilidades de enterramiento y las condiciones ecológicas que promueven la probabilidad de ocurrencias de este tipo de mortalidad y su importancia para las poblaciones humanas en términos de la generación de un nicho carroñero.

\section{MORTANDAD POR ESTRÉS INVERNAL EN LA PATAGONIA}

Se dispone de numerosos datos en la provincia de Santa Cruz sobre muertes de animales por estrés invernal, en especial en el caso de los ovinos, debido a las pérdidas que estas catástrofes provocan en el negocio agropecuario. En efecto, las grandes nevadas han sido un factor de riesgo para la producción ovina a lo largo del siglo XX. Childs (1997), por ejemplo señala en su libro El J immy. Bandido de la Patagonia: (En el invierno de 1905)... las tormentas continuaron por cuatro días y cuatro noches sin parar. A Magan de catorce mil le quedaron sólo ciento sesenta vivas en el recuento final. A la estancia La Esperanza le quedaban doce mil de las treinta mil que se habían contado a la entrada del invierno. Sturzenbaum y Borreli (2001) señalan que las nevadas de 1973 y 1994 también provocaron grandes pérdidas, pero que aún peores han sido la ya citada de 1905 y la de 1995. Desde el punto de vista histórico, las mayores pérdidas han ocurrido en campos cercanos a la cordillera o en aquellos que superan los 400 metros de altitud. Se han calculado tasas de mortandad para borregos entre 5 y $80 \%$, para ovinos adultos entre 5 y $95 \%$, en bovinos entre 5 y $50 \%$ y para equinos entre 5 y 70\% (Sturzenbaum y Borrelli 2001). Estas son tasas de mortalidad muy altas, y cabe aclarar que en el caso de las ovejas puede ser inferior que en poblaciones de animales salvajes, debido a que los ovinos se agrupan en círculo ("montoneras") para no perder calor, y son animales muy generalistas en su alimentación, pudiendo sobrevivir bajo la nieve hasta 15 días, contándose casos de supervivencia luego de 30 días enterrados (ver foto 13-3 en Sturzenbaum y
Borrelli 2001). La causa de muerte varía con la duración e intensidad de las nevadas: en el caso de nevadas tempranas y con "voladeros" de nieve, la mortalidad se produce por la sofocación que ocurre en las llamadas "montoneras". Cuando la presencia de nieve se mantiene por demasiado tiempo, la muerte se produce por estrés e inanición (Sturzenbaum y Borrelli 2001).

Entre los camélidos sudamericanos, las causas de muerte más comúnmente mencionadas son depredación, caza, enfermedades, sequía, accidentes, rayos, y estrés invernal. Numerosos autores señalan como causa importante de fluctuaciones en las poblaciones de guanacos el estrés invernal (Raedeke 1976; Merino y Cajal 1993; Cajal y Ojeda 1994; B orrero 2001). A juzgar por la literatura de viajeros y exploradores que recorrieron la Patagonia a fines del siglo XIX y principios del XX, los episodios de mortalidad catastrófica de guanacos son muy frecuentes. En efecto, podemos hallar referencias a estos episodios en Fitz Roy (1839), Darwin (1951:195-196), H udson (1953: 277), G onzález (1965:66), Vignati (en Mendoza 1965:40, nota 66), Moreno (1969 [1879]: 263 y 264), Casamiquela (1983), Musters (1997 [1879]: 71-72), Madsen (1998:38), Prichard (2003 [1903]: 142-143, 193-195, 199, 246 y 264) y Hatcher (2003 [1903]:297), por ejemplo, señala refiriéndose al invierno de 1905 en el suroeste de la provincia de Santa Cruz: En ese mismo invierno vi perecer decenas de millares de guanacos dentro de un radio de pocos kilómetros. Todos acudían al río en busca de matas, pero al no encontrarlas se amontonaban y morían, y los vivos se trepaban sobre los muertos hasta formar grandes pirámides; algunas de estas semejaban bolsas de trigo y debían contener más de dos mil animales cada una. Los sobrevivientes estaban tan mansos que caminábamos entre ellos como en un corral de ovejas y teníamos que apartarlos para avanzar.

Las condiciones ecológicas capaces de generar estos conjuntos son el resultado de parámetros normales en los ecosistemas del sur patagónico. Como ya fuera reseñado, estos episodios de mortalidad masiva no son excepcionales, ya que en años de inviernos duros miles de animales mueren. Así, la heterogeneidad y la imprevisibilidad son características que definen el clima del sur de Patagonia (Endlicher y Santana Aguila 1988, 1997, Sturzenbaum y Borrelli 2001). En el área, las cadenas tróficas están caracterizadas por una baja diversidad de especies dentro 
de cada microambiente (Pisano 1989-1990), conforme a la expectativa ecológica que predice un descenso de la biodiversidad con el aumento de la latitud, mejor conocida como Regla de Rapoport (Dynesius y Jansson 2000, Sax 2001). Esta baja diversidad tiene como consecuencia una baja redundancia funcional (sensu Tilman et al. 1997, Waide et al. 1999): los nichos disponibles son ocupados por una, o a lo sumo dos especies. Una implicación de esto es que cualquier fluctuación en la productividad de una especie tiene un efecto amplificado en el ecosistema total (Pisano 19891990). Las oscilaciones en la productividad vegetal se reflejan en las poblaciones de animales. El resultado es que la capacidad de sustentación de la región puede variar enormemente de año en año. Hay dos factores que producen fluctuaciones en las poblaciones de ungulados. Por un lado la calidad de las pasturas de verano, regulada por las precipitaciones de invierno y primavera, que en caso de no ser óptima contribuye a una baja supervivencia invernal y a una mayor predisposición a parasitismo y enfermedad (Sturzenbaum y Borrelli 2001), y por el otro el forraje invernal reducido. Es decir que períodos de sequías prolongados, muy comunes en el área de estudio (Endlicher y Santana Aguila 1988) generan condiciones propicias para este tipo de fenómenos. Por otra parte, la abundancia y calidad del forraje invernal, aún sin fluctuaciones en las pasturas veraniegas, afecta ciertos parámetros de productividad poblacional, como edad de maduración sexual, concepción y supervivencia de crías, longevidad promedio y resistencia a las enfermedades, los parásitos y los predadores. En el caso del guanaco, se ha observado que las condiciones de mortalidad de chulengos son más altas durante los primeros siete meses de vida, y que en invierno la mortalidad aumenta aproximadamente un $6 \%$ con cada centímetro de nieve caída (Sarno et al. 1999). En condiciones de alta precipitación invernal, como el caso del invierno de 1995 (Endlicher y Santana Aguila 1997), la acumulación de hielo y nieve sobre la vegetación lleva al hambre y a un incremento de la mortalidad (Montes et al. 2000). Sumado a ello la reducida nutrición maternal disminuye el peso al nacer de las crías y su tasa de supervivencia. Censos realizados en diferentes ambientes de Tierra del Fuego luego de un invierno con grandes nevadas (1995) han permitido calcular casi un $26 \%$ de mortalidad asociada con estos factores climáticos extremos (Montes et al. 2000).

\section{ESTRUCTURA POBLACIONAL DEL GUANACO}

El guanaco es un herbívoro generalista, básicamente pastador pero que también puede ramonear de acuerdo a la oferta de alimento disponible y a la época del año. Preferentemente habita lugares abiertos, ocupando sectores de estepa o pradera, aunque también puede hacer uso de hábitat de bosque (Borrero 2001, Cajal y Ojeda 1994, Montes et al. 2000). Su comportamiento puede describirse como estacionalmente territorial, aunque con excepciones, relacionadas fundamentalmente con la presencia de zonas con disponibilidad de agua (Casamiquela 1983, Oporto 1983).

La ocupación de entornos rigurosos y cambiantes obliga a esta especie a adaptarse mediante modificaciones en la composición, tamaño y proporciones de los distintos grupos sociales. En este sentido, numerosos autores (ver revisión en Puig y Videla 1995) han destacado las variaciones estacionales en estos parámetros y las diferencias entre poblaciones. En las poblaciones silvestres de guanacos pueden diferenciarse tres estructuras básicas: grupos familiares, grupos de machos e individuos solitarios. Los primeros están conformados por un macho adulto con hembras y sus crías. Estas últimas se hallan estrechamente asociadas a sus madres y se mantienen en el grupo hasta que alcanzan los 10 a 15 meses de edad (Franklin 1983, Larrieu et al. 1983). Estos grupos familiares presentan un orden jerárquico claro, en el cual el macho (relincho) manifiesta actitudes de dominancia sobre las hembras y las crías. Estos grupos en general están conformados por un número variable de animales (5 a 13 integrantes), con un promedio de 8,2 individuos, entre los cuales se diferencian un macho adulto, 5,6 hembras $(S D=1,4)$ y 2,9 crías $(S D=1,4)$ (Puig y Videla 1995). En los grupos de machos, el rango de edad es más variado y no hay comportamiento de liderazgo marcado. No se han observado relaciones de dependencia entre animales maduros y juveniles. EI tamaño de estas agrupaciones es algo mayor que el relevado para los grupos familiares ( 7 a 20 individuos), promediando los 10 animales $(S D=4,64)$ (Puig y Videla 1995). Finalmente, los individuos solitarios han sido descriptos como animales física y sexualmente maduros, que presentan el impulso de unirse a otros (Oporto 1983).

$\mathrm{H}$ ay otras estructuras descriptas en algu- 
nas poblaciones: grupos familiares sin machos, y grupos mixtos. En el caso de los grupos familiares sin machos, se trataría de hembras juveniles 0 adultas con o sin crías, que abandonan su territorio, posiblemente debido a la expulsión de los juveniles por parte del relincho. Todos los autores que han identificado este tipo de agrupaciones (Franklin 1982, Merino 1988, Merino y C ajal 1993, O rtega 1985) señalan el carácter transitorio de las mismas, dado que terminan fusionándose con un grupo familiar ya existente o formando uno nuevo al unirse a un macho solitario; serían estructuras sociales transicionales desde su territorio a los grupos mixtos y viceversa. EI promedio de estos grupos es de 2,6 animales $(S D=0,5)$, conformados por hembras y crías en proporciones similares (Puig y Videla 1995). Los grupos mixtos, por su parte, consisten en agregaciones de varios tipos de grupos sociales, que se forman hacia fines del otoño y migran hacia las áreas de invierno, separándose al aproximarse la época de reproducción (Puig 1986, Puig y Videla 1995). El tamaño de los grupos mixtos es muy variable, pudiendo llegar hasta los 150 individuos (Franklin 1982), aunque las estima- ciones disponibles arrojan valores promedio menores, del orden de los 38,7 individuos en $\mathrm{La}$ Payunia y de 25,3 integrantes para Torres del Paine (Puig y Videla 1995).

\section{SITIO ALERO LOS GUANACOS 1}

El sitio Alero Los Guanacos 1 se ubica al oeste del lago Cardiel, en la estancia Cerro Bayo ( $48^{\circ} 48^{\prime} 42,4^{\prime \prime} \mathrm{S}, 71^{\circ} 23^{\prime} 39,1^{\prime \prime}$ O), a 385 msnm (Fig. 1), sobre el sector denominado Cañadones (Goñi et al. 2000). Los restos de guanacos relevados se encuentran junto a un gran paredón de arenisca, muy friable, perteneciente a la formación Lago Cardiel (Ramos 1982). El paredón tiene una orientación noroeste y en algunos sectores presenta una visera, que conforma un alero, que otorga cierta protección contra la lluvia y la nieve.

En el talud del sitio se registró abundante material arqueológico en superficie: desechos de talla, raederas, molinos y preformas de bolas. L a materia prima más representada es la limolita, seguida por el sílice, la obsidiana y la diabasa.

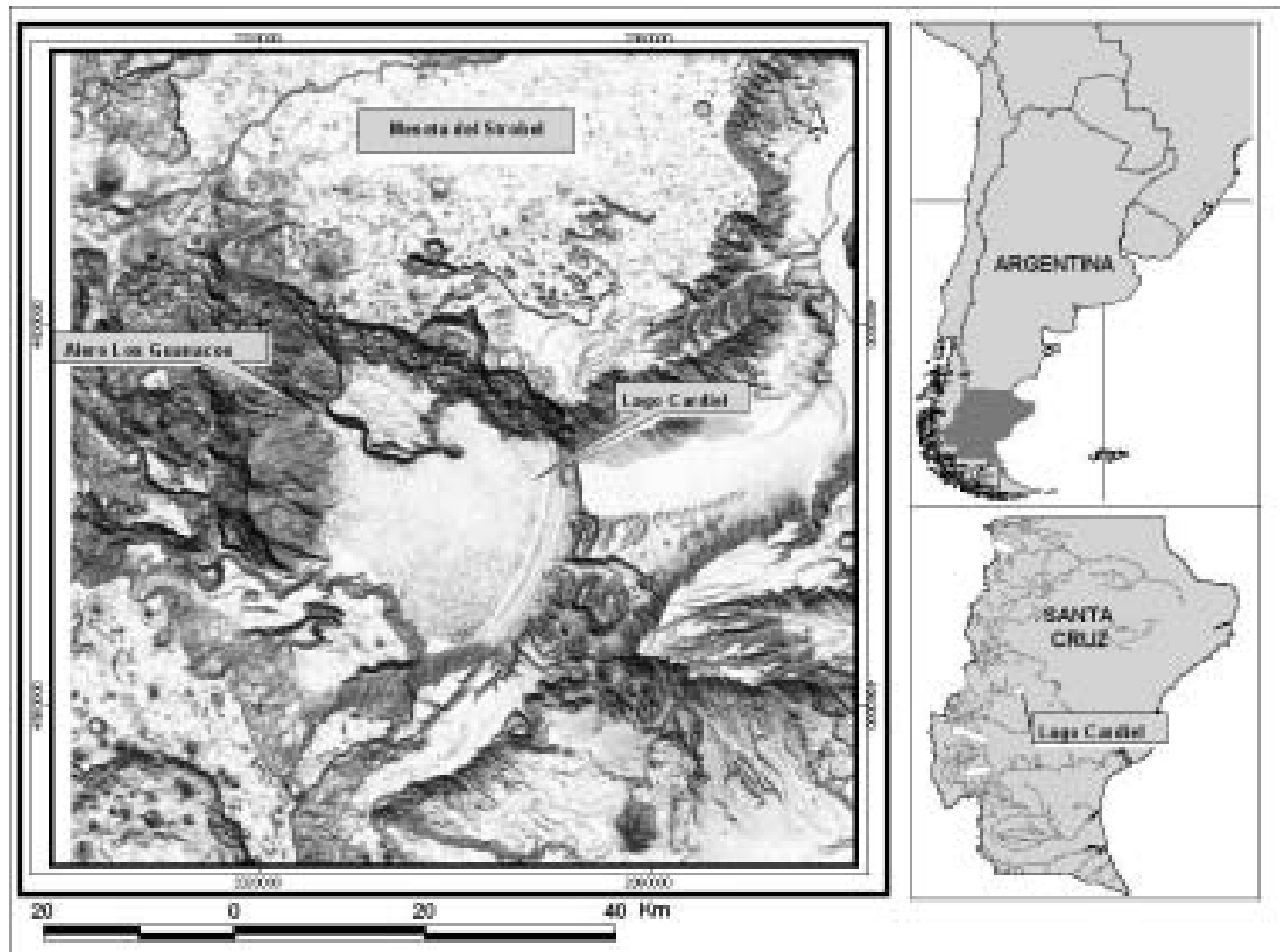

Fig. 1. Ubicación del Alero Los Guanacos 1. 


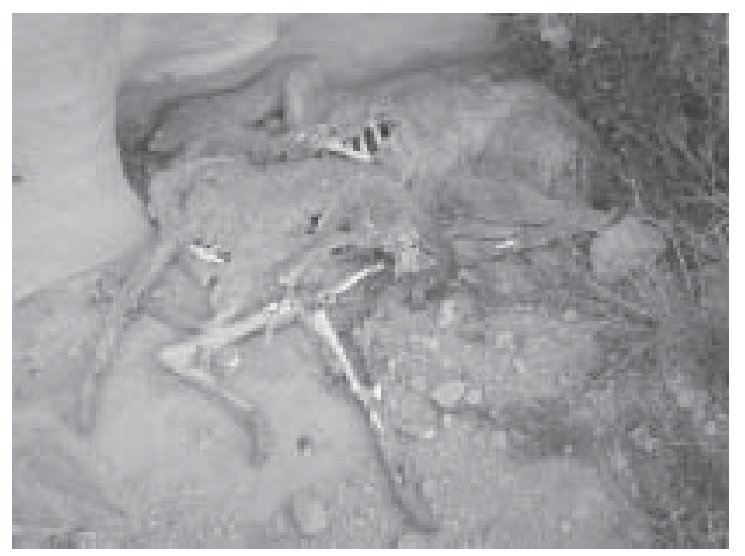

Fig. 2. Asociación entre individuos.

El conjunto faunístico

En el sitio Los Guanacos 1 se observó en febrero de 2005 la presencia de 36 carcasas de guanaco. Asimismo, en el farallón contiguo se observaron los restos de al menos 16 animales más.

En el curso del relevamiento se tomaron medidas de la concentración de carcasas (largo y ancho de la misma) y se realizaron plantas en papel milimetrado para dar cuenta de la distribución espacial de los restos. Asimismo se tomaron fotografías de los diferentes individuos. Para cada uno de ellos se consideraron las siguientes variables: estado de conservación (presencia de cuero, tejidos, contenido estomacal y estadio de meteorización), categoría de edad, posición de muerte, grado de enterramiento, grado de articulación, actividad de carnívoros, pisoteo y la asociación entre individuos y artefactos líticos. Se añadieron notas adicionales acerca de la orientación general del conjunto, características del sedimento que forma el sustrato del alero y presencia de huesos depositados previamente en el sitio (sobre la base de estadios de meteorización diferencial con respecto a los de la concentración considerada). Se recolectó una muestra de hue- sos que presentaban un patrón de fractura particular para ser analizadas en el laboratorio. Por último, como fuera observada la presencia de huesos sueltos contra la pared del alero, se relevaron los mismos a lo largo de 8 metros.

Los animales se hallan concentrados sobre la pared del alero, distribuidos a lo largo de aproximadamente 50 metros y sobre una franja de 6 metros de ancho. La orientación del conjunto es noroeste. La distribución de carcasas es discontinua, generándose grupos contiguos de muertes (Fig. 2). Sobre la base de la proximidad de carcasas y elementos se definieron tres concentraciones de individuos (Tabla 1).

$L$ as concentraciones, en general separadas por la presencia de vegetación y rasgos topográficos propios del alero como bloques 0 desniveles en el piso, sugirieron la posibilidad de un episodio de mortalidad en masa debido a alguna catástrofe natural. EI Sr. Arturo Olivero, empleado de la estancia L a Carlina, que conocía el conjunto, indicó que se formó durante las fuertes nevadas del año 2000 , es decir que el conjunto tenía 5 años en el momento en que fuera relevado. La mortalidad provocada por estas nevadas no se circunscribió a la concentración del Alero Los Guanacos 1, sino que numerosos animales murieron a lo largo de las bardas de los ríos cercanos, conservando asimismo una alta integridad (articulados, con cuero y contenido estomacal). La Tabla 2 resume los valores de las variables analizadas para cada una de las concentraciones y para cada individuo, las cuales serán tratadas a continuación.

\section{Estado de conservación}

Todos los animales presentan cuero, pelo, tejidos blandos y ligamentos (Fig. 3). También se preservaron los músculos y los órganos de la cavidad abdominal y el contenido estomacal. Bajo condiciones climáticas de humedad y temperaturas moderadas, la degradación de tejidos blandos procede desde "dentro" debido a la acción

TABLA 1. Concentraciones.

\begin{tabular}{|c|c|c|c|}
\hline Concentración & Frecuencia de individuos & Superficie $\left(\mathrm{m}^{2}\right)$ & Distancia entre concentraciones $(\mathrm{m})$ \\
\hline 1 & 4 & 10 & - \\
2 & 11 & 60 & 2 (entre 1 y 2) \\
3 & 21 & 230 & 6 (entre 2 y 3) \\
\hline
\end{tabular}


TABLA 2: Información general sobre el conjunto.

\begin{tabular}{|c|c|c|c|c|c|c|c|}
\hline $\begin{array}{c}\text { Concen } \\
\text { tración / } \\
\text { Individuo }\end{array}$ & $\begin{array}{c}\text { Categoría } \\
\text { de Edad }\end{array}$ & $\begin{array}{c}\text { Enterra- } \\
\text { miento }\end{array}$ & $\begin{array}{l}\text { Posición } \\
\text { de muerte }\end{array}$ & Articulación & $\begin{array}{l}\text { Acción de } \\
\text { Carnívoros }\end{array}$ & $\begin{array}{l}\text { Asociación } \\
\text { entre } \\
\text { individuos }\end{array}$ & $\begin{array}{l}\text { Asociación } \\
\text { con } \\
\text { artefactos }\end{array}$ \\
\hline $1 / 1$ & J uvenil & No & Acostado & $\mathrm{Si}$ & No & No & No \\
\hline $1 / 2$ & J uvenil & No & Sentado & $\mathrm{Si}$ & No & No & $\mathrm{Si}$ \\
\hline $1 / 3$ & Adulto & No & Acostado & $\mathrm{Si}$ & No & No & $\mathrm{Si}$ \\
\hline $1 / 4$ & Adulto & No & Acostado & No & Sí & No & No \\
\hline $2 / 5$ & Adulto & No & Sentado & $\mathrm{Si}$ & No & No & $\mathrm{Si}$ \\
\hline $2 / 6$ & J uvenil & No & Sentado & No & No & $\mathrm{Si}$ & No \\
\hline $2 / 7$ & Adulto & No & Sentado & $\mathrm{Si}$ & No & $\mathrm{Si}$ & No \\
\hline $2 / 8$ & Adulto & No & Sentado & Si & No & Si & No \\
\hline $2 / 9$ & J uvenil & No & Acostado & Si & No & $\mathrm{Si}$ & No \\
\hline $2 / 10$ & Cría & No & Sentado & $\mathrm{Si}$ & No & No & No \\
\hline $2 / 11$ & J uvenil & No & Sentado & $\mathrm{Si}$ & No & No & No \\
\hline $2 / 12$ & Adulto & No & - & No & Sí & No & No \\
\hline $2 / 13$ & Cría & Sí & Acostado & $\mathrm{Si}$ & No & No & No \\
\hline $2 / 14$ & Adulto & No & - & No & Sí & No & No \\
\hline $2 / 15$ & J uvenil & Sí & Acostado & $\mathrm{Si}$ & No & No & $\mathrm{Si}$ \\
\hline $3 / 16$ & J uvenil & No & Sentado & $\mathrm{Si}$ & No & $\mathrm{Si}$ & No \\
\hline $3 / 17$ & Adulto & No & Sentado & $\mathrm{Si}$ & No & $\mathrm{Si}$ & No \\
\hline $3 / 18$ & J uvenil & No & Acostado & $\mathrm{Si}$ & No & $\mathrm{Si}$ & No \\
\hline $3 / 19$ & Adulto & Sí & Sentado & $\mathrm{Si}$ & No & $\mathrm{Si}$ & No \\
\hline $3 / 20$ & Cría & No & Acostado & $\mathrm{Si}$ & No & $\mathrm{Si}$ & No \\
\hline $3 / 21$ & J uvenil & No & Sentado & $\mathrm{Si}$ & No & $\mathrm{Si}$ & No \\
\hline $3 / 22$ & Cría & No & Acostado & $\mathrm{Si}$ & No & No & No \\
\hline $3 / 23$ & J uvenil & No & Acostado & $\mathrm{Si}$ & No & No & No \\
\hline $3 / 24$ & Adulto & No & Sentado & $\mathrm{Si}$ & No & No & $\mathrm{Si}$ \\
\hline $3 / 25$ & J uvenil & No & Acostado & $\mathrm{Si}$ & No & $\mathrm{Si}$ & No \\
\hline $3 / 26$ & Adulto & No & Sentado & $\mathrm{Si}$ & No & $\mathrm{Si}$ & No \\
\hline $3 / 27$ & Cría & No & Sentado & $\mathrm{Si}$ & No & No & No \\
\hline $3 / 28$ & J uvenil & No & Acostado & $\mathrm{Si}$ & No & $\mathrm{Si}$ & No \\
\hline $3 / 29$ & Adulto & No & Sentado & $\mathrm{Si}$ & No & $\mathrm{Si}$ & No \\
\hline $3 / 30$ & Adulto & No & Sentado & $\mathrm{Si}$ & No & No & No \\
\hline $3 / 31$ & J uvenil & No & Acostado & $\mathrm{Si}$ & No & No & No \\
\hline $3 / 32$ & J uvenil & No & Acostado & $\mathrm{Si}$ & No & $\mathrm{Si}$ & No \\
\hline $3 / 33$ & Cría & No & Acostado & No & No & $\mathrm{Si}$ & No \\
\hline $3 / 34$ & Cría & No & Acostado & $\mathrm{Si}$ & No & $\mathrm{Si}$ & No \\
\hline $3 / 35$ & Adulto & No & Sentado & $\mathrm{Si}$ & No & $\mathrm{Si}$ & No \\
\hline $3 / 36$ & Adulto & No & Acostado & $\mathrm{Si}$ & No & No & No \\
\hline
\end{tabular}

de microorganismos entéricos y desde "fuera" a través de la colonización por parte de microorganismos del suelo e insectos (Micozzi 1991). De esta forma, la descomposición debida a la actividad bacteriana es acelerada en ambientes con rangos de temperatura entre los 15 y 370 C, y la desecación debe ser rápida y casi completa para que se preserven tejidos blandos. Conforme decrece la temperatura, el grado de desecación requerido para la preservación se reduce en gran medida. De esta manera, con tempera- turas inferiores a los $50 \mathrm{C}$, el grado de desecación debe ser mínimo, y por debajo de los 0 ㅇ $C$, no se requiere ningún tipo de desecación, dado que el congelamiento es la forma de preservación más efectiva. Asimismo, el congelado previo también retarda la actividad bacteriana luego de que los tejidos sean descongelados a temperatura ambiente (Micozzi 1986, 1991). En este sentido, animales que han estado sujetos a procesos de congelamiento y posterior descongelamiento parecen descomponerse desde "afuera", siendo el 


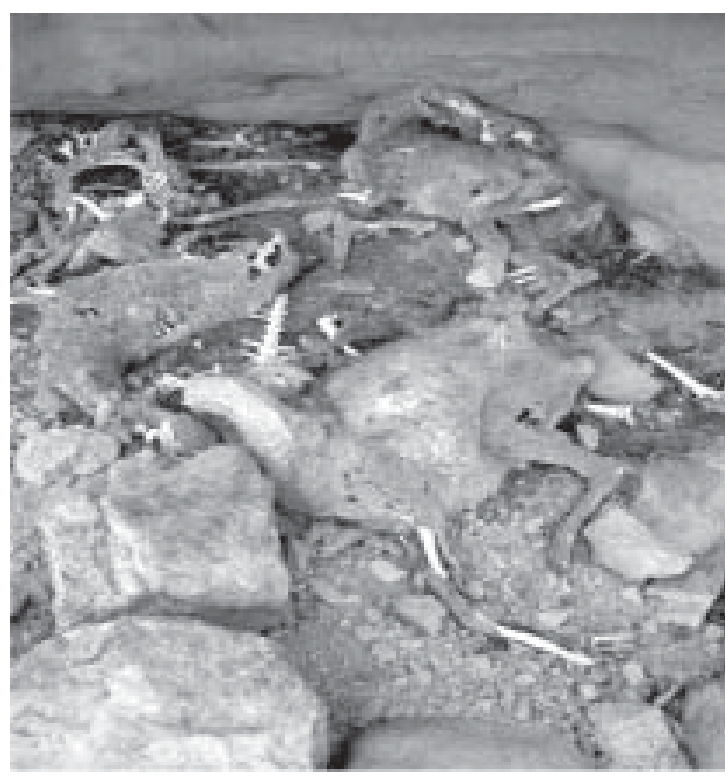

Fig. 3. Estado de conservación de los individuos.

deterioro debido a la invasión por parte de organismos externos del suelo, mientras que animales no congelados se descomponen desde "dentro" debido a la diseminación de microorganismos entéricos.

El estado de conservación de las carcasas del sitio es excepcional, considerando que mediaron 5 años desde que se formó el conjunto hasta que fue relevado. Hay varios factores que podrían dar cuenta del estado de preservación de los restos: a) condiciones ambientales del área de estudio, como bajo porcentaje de humedad y alta radiación solar, sumado a la actividad eólica, que acelerarían la evapotranspiración ayudando a momificar los tejidos (Nasti 1994-1995), b) con- diciones de congelamiento y posterior descongelamiento de los tejidos, que inhibirían o retardarían en gran medida el proceso de putrefacción (Micozzi 1986) y c) una combinación de ambos factores. Independientemente de cuál de estos factores u otros haya tenido más peso a la hora de explicar el alto grado de conservación de los animales en el sitio, el resultado final es el mismo, dado que en todos los casos condujo a la momificación de los tejidos.

Las únicas porciones en las que en algunos casos se observa el hueso son la zona facial, las porciones distales de las extremidades (metapodios) y el área de la pelvis y el abdomen. Los huesos expuestos se presentan blanqueados y muy poco meteorizados (estadio 1 sensu Behrensmeyer 1978). Esto concuerda con observaciones realizadas en otros ambientes, como la Puna (Nasti 1994-1995), que indican que la presencia de cuero protege a los huesos de la degradación por meteorización.

\section{Estructura etaria}

La edad de los animales fue definida a partir del tamaño general de las carcasas y el estadio de fusión de los huesos largos, la escápula y la pelvis. Sobre la base de estos criterios, se definieron tres grupos de edad: crías, juveniles y adultos. En el caso de las crías, el tamaño de las carcasas y la presencia del coracoides de la escápula e isquium-ilium-pubis de la pelvis no fusionado permitió inferir que se trataba de animales menores a un año (Kaufmann 2004). Fueron considerados juveniles aquellos animales que presentaban huesos largos sin fusionar, proceso que en los guanacos se completa entre los 30 y 45 meses (Ibid). Finalmente, la categoría de adultos

TABLA 3: Estructura etaria de poblaciones de guanaco estudiadas (porcentuales considerando dos clases de edad, crías y adultos + juveniles) (Modificado a partir de Saba et al. -1995).

\begin{tabular}{|l|c|c|c|}
\hline \multicolumn{1}{|c|}{ Localidad } & \% Crías & $\begin{array}{c}\text { \% J uveniles } \\
\text { adultos }\end{array}$ & Referencias \\
\hline R. La Payunia (Mendoza, Argentina) & 21,4 & 78,6 & Puig 1986 \\
Depto. Collón Curá (Neuquén, Argentina) & 29,7 & 70,3 & Gader y Del Valle 1982 \\
Ea. Don Carlos (Chubut, Argentina) & 7,8 & 92,2 & De Lamo et al.1982 \\
Ea. Don Carlos (Chubut, Argentina) & 6,8 & 93,2 & Saba 1987 \\
Península Mitre (Tierra del Fuego, Argentina) & 10 & 90 & Merino y Cajal 1993 \\
Zona Central (Tierra del Fuego, Argentina) & 16,7 & 83,3 & Bonino 1988 \\
Sitio Los Guanacos 1 (Santa Cruz, Argentina) & 19,4 & 80,54 & Este trabajo \\
\hline
\end{tabular}


está conformada por animales con fusión completa. Todos los grupos de edad están representados en el conjunto (Tabla 2). Como se puede observar en la Tabla 3, la estructura etaria del sitio es compatible con las relevadas en otras poblaciones silvestres de guanacos.

Posición de muerte

En la mayoría de los individuos fue posible observar la posición de muerte (Tabla 2). Los animales se hallaban principalmente en dos posiciones: reclinados sobre el costado derecho o izquierdo del cuerpo con las extremidades extendidas al costado del tórax ("acostado" o en posición de reclinación lateral, sensu Haynes 1995) o descansando sobre el área ventral, con las extremidades flexionadas debajo del cuerpo ("sentado" o en posición de reclinación esternal, sensu Clubb y Macdonald 2004). En el caso del Alero Los Guanacos 1, una posición parece ser tan común como la otra, como se observa en la tabla 2. Esto está en concordancia con los datos de Haynes (1995) acerca de la posición de muerte de camellos silvestres en Australia. De Lamo (1995) ha observado que la pérdida de calor debido a la exposición a vientos intensos se ve reducida en un $60 \%$ en el caso de guanacos que adoptan la posición de sentado. Por otra parte, este autor señala que la tasa metabólica basal es un $66 \%$ más alta en un individuo parado o caminando lentamente que en un animal echado. Es interesante notar que la posición de reclinación esternal también es la adoptada en el caso de animales sometidos a anestésicos para facilitar su captura (Clubb y Macdonald 2004). Esto indica que esta posición es adoptada comúnmente por los animales para reducir los costos energéticos asociados con el desplazamiento y la pérdida de calor por convección forzada de vientos intensos, así como en momentos de estrés intenso. Aproximadamente un $25 \%$ de los animales mostraba contracción post-mortem de los ligamentos del cuello. Esta posición del cuello también ha sido señalada para el caso de camellos silvestres (Haynes 1995).

\section{Grado de enterramiento}

Las carcasas apoyan sobre un sedimento conformado por fragmentos de la pared que forma la roca base del alero y por una capa de guano de oveja y de guanaco. Además se observan huesos sueltos con estadios de meteorización en general avanzados, que corresponden mayormente a oveja y guanaco. O bservaciones realizadas en áreas cercanas como Lago Argentino (Borrero 2001) indican que la acumulación de capas de guano inhibe en gran medida las posibilidades de enterramiento de huesos. Ese parece ser el caso del Alero Los Guanacos 1. Esto es importante, porque antes de la introducción de la ganadería ovina el enterramiento podría haber sido más rápido. Más allá de este factor retardatorio, unos pocos individuos se hallan en proceso de enterramiento. Otras dos variables importantes en relación con este proceso son, por un lado, la presencia de vegetación y, por otro, la existencia de rasgos topográficos propios del sitio como desniveles, que facilitan el proceso de sepultamiento al generar aporte de sedimentos. Es el caso, por ejemplo, de los individuos 13 y 15 , que se hallan al pie de una pendiente.

Es interesante notar la presencia continua de huesos sueltos en distintos grados de enterramiento junto a la pared trasera del alero. En la Tabla 4 se presenta a modo de ejemplo la distribución de huesos a lo largo de ocho metros que se asocia con la concentración 2. Sólo se relevaron huesos de guanaco en orden sur-norte, de acuerdo al grado de proximidad con la pared del alero. De su observación surgen algunas tendencias: el esqueleto axial se halla representado mayoritariamente por cráneo-mandíbula y axis, mientras que el esqueleto apendicular por la extremidad delantera superior (escápula, húmero y radioulna) y por las extremidades delantera y trasera inferior (metacarpo, metatarso y falanges). De acuerdo con Nasti (1994-1995:74) el cuero se retrae lentamente de la zona ventral a la dorsal y del área del cráneo a la caudal. Borrero (1988a), por su parte, resume la secuencia de desarticulación de guanacos de la siguiente manera: 1) cráneo y atlas, cola, 2) pata delantera, 3) costillar, 4) esqueleto axial, pata trasera. Es decir que en general los huesos representados corresponden a aquellas zonas donde el cuero se retira más rápidamente 0 donde la articulación es más débil: cráneo y cuello, extremidad delantera y porciones distales de las extremidades.

Grado de articulación, acción de carnívoros y pisoteo

En otros estudios se ha observado que la pérdida de cuero es un factor desencadenante 
TABLA 4: Elementos sueltos de guanaco ubicados contra el borde de la pared del alero.

\begin{tabular}{|c|}
\hline Elemento \\
\hline Cráneo \\
\hline Mandíbula \\
\hline Escápula \\
\hline Radioulna \\
\hline Metatarso \\
\hline Húmero \\
\hline 1o falange \\
\hline Húmero \\
\hline Húmero ds. \\
\hline Húmero px. \\
\hline Hemipelvis \\
\hline Fémur \\
\hline Metacarpo hasta pezuña \\
\hline Axis \\
\hline Hemipelvis \\
\hline Metacarpo \\
\hline
\end{tabular}

de la desarticulación (Borrero 1988a). Toots (1965) ha señalado que esto es particularmente cierto cuando la desecación ocurre antes de la descomposición, debido a que hay poco tejido asociado. La conservación de cuero, por otra parte, es mayor cuando se dan condiciones de bajas temperaturas, por lo que el invierno sería un factor retardatorio de este proceso (Borrero 1988a, Haynes 1982). Como ya fuera señalado, en el conjunto del Alero Los Guanacos 1 la mayoría de los animales conserva el cuero y ligamentos en buenas condiciones, y este hecho posiblemente explica que la mayoría de los individuos se encuentren articulados (Tabla 2). Los dos tipos de posición de muerte tienen implicancias diferentes en cuanto al patrón de desarticulación. En este sentido, se espera un mayor grado de integridad en las carcasas de animales que murieron en posición de reclinación esternal, debido a que las extremidades se mantienen flexionadas debajo del tronco, es decir quedan "protegidas" por el esqueleto axial, que es la última parte en desarticularse (Borrero 1988a, Nasti 1994-1995).

En tres de los cinco individuos que se hallan desarticulados se relevaron marcas de carnívoros (ver Tabla 2). Estas carcasas son también las que muestran la mayor dispersión espacial. Esto es consistente con la observación de que la actividad de los carnívoros, aún los de pequeño tamaño, constituye una variable importante que acelera el proceso de separación de un esqueleto. Esto ocurre sustrayendo elementos durante la alimentación o dejando expuestos huesos individuales y zonas articulares, lo que favorece la desarticulación posterior.

El daño registrado se localiza en los procesos espinosos y las apófisis de las vértebras torácicas del individuo 4 , en los procesos transversos y espinosos de las vértebras del tórax del individuo 12 y el trocánter menor del fémur, la porción distal de la escápula, esternebras y porciones distales de las costillas del individuo 14. El tipo de daño no es muy intenso, compatible con el realizado por carnívoros de tamaño pequeño o mediano como zorros o perros. Los huesos afectados sugieren un procesamiento inicial de las carcasas, en la cual los carnívoros se interesan por las partes blandas como el estómago y posteriormente por la pata delantera (articulación escápula-húmero).

Aún cuando estas carcasas han sido perturbadas por carnívoros, la desarticulación en general sigue la secuencia dada por Borrero (1988a) y por Nasti (1994-1995) que a su vez muestran un alto grado de concordancia con la de Hill (1980) y de Binford (1981). Esto indica que la actividad de este agente ha sido suficiente para poner en marcha el proceso de dispersión de una carcasa, pero que no alteró a la misma de manera significativa. El carroñeo sólo afecta a individuos adultos.

Tal como fuera mencionado más arriba, se recolectó una muestra de huesos largos $(\mathrm{N}=3)$ para ser analizada en el laboratorio, debido a que presentaban un patrón de fractura particular. LoS huesos corresponden a los individuos desarticulados por la acción de carnívoros: fémur fracturado en dos segmentos, fragmento de húmero distal y tibia fracturada en cuatro segmentos. Tanto el fémur como la tibia se encuentran en el estadio 0 de meteorización, mientras que el húmero está en 1.

Las fracturas se dan en general en sentido longitudinal al eje mayor del hueso, con bordes frescos 0 astillados. No se relevó la presencia de hoyos de percusión y, aunque hay negativos, estos no presentan la forma de escotadura 
TABLA 5: Frecuencias de individuos por concentración, huesos y artefactos asociados.

\begin{tabular}{|ccc|}
\hline Concentración & Número de huesos & Artefactos asociados \\
\hline 1 (individuos 1 a 4) & 584 & 6 \\
2 (individuos 5 a 15) & 1606 & 3 \\
3 (Individuos 16 a 36) & 3066 & 22 \\
\hline Total & 5256 & 31 \\
\hline
\end{tabular}

semilunar que es característica de la fractura por percusión antrópica. Además, se observó la presencia de marcas de pisoteo (Behrensmeyer et al. 1986, Fiorillo 1989), las que no siguen una orientación determinada y son, en general, superficiales. Si bien algunas son más profundas y morfológicamente resultan similares a las marcas de corte, no muestran una posición anatómica que tenga sentido en términos de procesamiento ya que se ubican en las diáfisis y son transversas al eje mayor del hueso. Aquí es evidente que la pérdida del cuero ha expuesto las superficies de los huesos a condiciones subaéreas, poniéndolas en contacto con la matriz sedimentaria. Esto ha favorecido tanto el surgimiento de estadios de meteorización incipiente como la fractura y aparición de marcas de pisoteo, provocadas posiblemente por la reutilización del alero por parte de guanacos y ovejas. Por último, el fémur muestra evidencias de marcas de roedor principalmente en torno a los bordes de fractura.

Asociación entre individuos y artefactos

Dentro del conjunto, varios individuos se presentaban en estrecha asociación espacial (Fig. 2). La mayoría de estos animales presenta los cuellos entrecruzados y, menos comúnmente, el cuello de uno apoya sobre el cuerpo de otro. De todos los subconjuntos de este tipo relevados, ocho de nueve corresponden a parejas. La excepción la constituye el conjunto formado por los individuos 16, 17 y 18 por un lado, y el 18 asociado con el individuo 19. Notoriamente, en todos los casos la asociación se produce entre individuos pertenecientes a diferentes grupos etarios: juvenil - adulto (6 casos), juvenil - cría (2 casos) y cría - adulto (1 caso). Esta asociación podría interpretarse como grupos de hembras con sus crías / juveniles, sin embargo, no se cuenta hasta el momento con los datos acerca del sexo de los animales.

La distribución de materiales arqueoló- gicos líticos de superficie es relativamente continua sobre la superficie del alero y presenta una baja densidad. Aproximadamente un 14\% de las carcasas se hallaba asociado con artefactos líticos (Tabla 2). En algunos casos el material es muy abundante: a modo de ejemplo, alrededor del individuo 15 hay 12 lascas de limolita, una raedera de dacita negra, una lasca de obsidiana y 3 lascas de dacita (relacionadas con la manufactura de bolas de boleadora). En la Tabla 5 se presentan las frecuencias de animales presentes en cada concentración, el potencial de huesos que cada una de ellas representa (obtenido al multiplicar el número de animales por el número de huesos presente en un guanaco) y la de artefactos asociados.

\section{DISCUSIÓN Y CONCLUSIONES}

A lo largo de este trabajo se presentó información tafonómica derivada de observaciones realizadas en el sitio Alero Los Guanacos 1. Dichas observaciones son concordantes con un evento de mortalidad masiva de guanacos provocado por estrés invernal en el año 2000. Las evidencias para sustentar un evento de muerte sincrónica son: la relación de proximidad espacial entre los individuos, la presencia de cuero y contenido estomacal en todas las carcasas relevadas, así como de estadios de meteorización similares en las porciones expuestas de los huesos. Estas dos últimas características muestran la alta integridad del conjunto, aún cuando se haya relevado la acción de carnívoros y la presencia de marcas de pisoteo. Además, la estructura etaria, la cantidad de animales presentes y la asociación espacial entre individuos sugiere que un grupo mixto o varios grupos familiares de adultos, juveniles y crías murieron juntos.

El conjunto del Alero Los Guanacos 1 posee algunas características en común con procesos de mortalidad de guanacos producidos por factores no relacionados con situaciones de mor- 
talidad catastrófica. Así, muchos de los criterios definidos por Borrero (1989) y por L'Heureux y Borrero (2002) para el reconocimiento de conjuntos producidos por seres humanos frente a los derivados por otros agentes son pertinentes en este caso. Las observaciones aquí realizadas permiten postular los siguientes criterios adicionales para conjuntos masivos provocados por situaciones de estrés invernal y, si bien ninguno de estos criterios por sí mismo es suficiente para definir que se está en presencia de un evento de mortalidad masiva, la aparición de varios de ellos en un conjunto debería informar acerca de este tipo de situaciones.

a) La presencia de gran cantidad de animales de una misma especie en un sitio. Esto podría indicar la existencia de cazas comunales, no obstante, los conjuntos óseos generados por seres humanos en Patagonia no presentan evidencias de ello; muy por el contrario, los MNI relevados en cada caso son bajos y presentan índices variables de fragmentación (Borrero 1990, Mengoni Goñalons 1995).

b) Un alto grado de superposición de elementos relacionado con las posiciones de muerte y el agrupamiento de los individuos producto de la búsqueda de protección contra el frío.

c) Presencia de carcasas completas y/0 alta abundancia de segmentos (extremidades, esqueleto axial) con alto grado de articulación.

d) Proporción axial /apendicular similar a la observada en individuos completos, o predominio de segmentos del esqueleto axial sobre el apendicular.

e) Alta proporción de elementos enteros.

f) Una estructura de sexo y edad consistente con la de segmentos determinados de una población de guanaco, tales como grupos mixtos, tropas de machos o grupos familiares.

Si bien la alta proporción de carcasas o elementos con estadios de meteorización homogéneos podría llegar a constituir un criterio adicional, se requiere un mayor tiempo de observación con el fin de corroborar su utilidad como tal.

¿Cuáles son las implicaciones de los eventos de mortalidad producto del estrés invernal en función de la arqueología de espacios restringidos como cuevas y aleros (que representa gran parte de los sitios sobre los cuales se basa la arqueología patagónica)? Es evidente que bajo cier- tas condiciones de estrés (v.g. condiciones ambientales crudas, enfermedades, vejez, etc.) los aleros son lugares propicios para que mueran animales porque ofrecen reparo. En otros sitios del noroeste de la provincia de Santa Cruz, como el Alero Destacamento Guardaparque y el Alero Dirección Obligatoria, ubicados en el Parque Nacional Perito Moreno (Goñi 1988), se ha detectado la presencia de animales muertos en la superficie de los mismos. Más aún, la mortalidad de animales no se circunscribe a guanacos, dado que en varios de estos sitios se han observado restos de ovejas (ver Cruz 1999, Tabla 2). Por otro lado, como es evidente en el caso del Alero Los Guanacos 1, la mortalidad de animales en este tipo de sitios ofrece potencial de mezcla con residuos producidos por la utilización por parte de los grupos humanos de estos mismos espacios, ya sea de restos de alimentación como de artefactos (Savanti et al. 2005). El resultado de esta mortalidad catastrófica es una verdadera lluvia de huesos (Tabla 5), aproximadamente 17,5 por metro cuadrado, yuxtapuesta con instrumentos líticos y huesos de diferentes taxones depositados previamente, en un espacio relativamente acotado de $300 \mathrm{~m}^{2}$. Los criterios recién enumerados alertan sobre el carácter tafonómico del conjunto óseo. Este tipo de situaciones también se ha observado en otros ambientes de Tierra del Fuego y Patagonia continental, indicando que pueden ser la norma más que la excepción en el registro arqueológico regional (Borrero 1988a y b, 2001, L'Heureux y Borrero 2002).

Se ha señalado que las cuevas y aleros tienen historias depositacionales complejas e idiosincráticas (Barton y Clark 1993, Straus 1990), implicando que hay mucha variabilidad en el potencial de enterramiento de restos. En el caso del Alero Los Guanacos 1, la presencia de una gruesa capa de guano de oveja inhibe en gran medida el enterramiento. Esta situación tiene implicaciones recién desde el momento de introducción de las ovejas. Sin embargo, determinadas condiciones como la presencia de vegetación y de rasgos topográficos del alero ya mencionados favorece este proceso en espacios acotados dentro del sitio y sus implicaciones si pueden considerarse con mayor profundidad temporal. Por otra parte, los huesos desarticulados que se ubican contra la pared trasera también tienen una alta probabilidad de enterramiento, porque la presencia de la pared hace que queden estables y porque es un espacio que actúa 
como trampa sedimentaria. Esto a su vez tiene implicancias con respecto al modelo de zonas drop y toss (Binford 1989), ya que en espacios restringidos como los mencionados se pueden formar acumulaciones análogas a una zona "toss" sin que medie intervención humana.

La importancia de este tipo de mortalidad en función de la subsistencia de las poblaciones que tenían al guanaco como su presa principal es evidente y diferente en el corto y largo plazo. En este sentido, los inviernos crudos generan una importante posibilidad de alimento en forma de animales aptos para consumo sin necesidad de cazarlos. En este punto, es interesante notar que, pese a lo que podría pensarse, la mayoría de los individuos que mueren de estrés invernal se hallan en buenas condiciones físicas. Cajal y Ojeda (1994) señalan que el $40 \%$ de las carcasas relevadas por ellos mostraba un grado de nutrición normal, el $27 \%$ un grado intermedio y sólo un $33 \%$ se hallaban mal nutridos. Además, la mortalidad de individuos no se produce azarosamente en el espacio, sino que es altamente concentrada en unos pocos sectores como aleros, cuevas y bardas de ríos. Por lo tanto, es un fenómeno estacional, repetitivo y con una localización espacial predecible, lo que lo hace muy interesante como fuente de recursos aprovechable a través de estrategias de carroñeo. A la vez, es una estrategia poco costosa y de bajo riesgo (Borrero et al. 2005) que emplea una oferta inmediata que se presenta en una escala regional. Por estas razones, la implementación del carroñeo por parte de las poblaciones cazadoras recolectoras no sólo sería esperable en momentos iniciales de poblamiento (Borrero et al. 2005), sino que pudo ser sistemáticamente utilizado a lo largo del tiempo 0, al menos, hasta la introducción del caballo. Esto último se sugiere dado que, conociendo la existencia del estrés invernal y su magnitud (ver Rockman y Steele 2003, Meltzer 2003), la posibilidad de abandono de espacios como respuesta al riesgo se habría visto favorecida en términos de la velocidad, distancia de desplazamiento y posibilidad de carga que brinda el caballo.

Se ha observado también que las fluctuaciones provocadas por esta variabilidad climática en la densidad y estructura de las poblaciones de ungulados son profundas en el largo plazo. Un evento de este tipo puede afectar la dinámica de una población por años (Alonso Aguirre et al. 1999, Berger 1983, Dumbar et al.
1999, Klein 1968, Martinka 1967, O 'H ara et al. 2001, Paterson etal. 1998, Reimers 1982, Schaller y J unrang 1988, entre otros). Esto no implica que las poblaciones de guanacos no se recuperen 0 que, en relación con lo recién presentado, los grupos humanos no cuenten con estrategias para hacer frente a este tipo de riesgos. Las poblaciones humanas emplean diferentes mecanismos para hacer frente a esta escasez de recursos (Halstead y O'Shea 1989, Minc y Smith 1989, Rowley-Conwy y Zvelebil 1989). En este sentido, una estrategia clásica implementada es la movilidad. En áreas bajas como el lago Cardiel, un descenso en la biomasa de animales podría disminuir en gran medida la productividad regional. Este podría ser un factor determinante en el uso discontinuo de la región por parte de las poblaciones cazadoras recolectoras. A la vez, cualquier tipo de recrudecimiento climático, como por ejemplo la "Pequeña E dad del Hielo" (1340-1660 A. D) (Villalba 1994), favorecería la ocurrencia de las condiciones planteadas. Esto tiene implicaciones que van más allá del estudio de un caso particular, considerando que el guanaco fue en gran medida la base de la subsistencia de los grupos cazadores recolectores en Patagonia Meridional.

\section{AGRADECIMIENTOS}

A Silvana Espinosa, Florencia Gordón, María Gutiérrez, Luis Borrero, Gustavo Martínez y Mariano Merino, al igual que a los dos evaluadores, por la lectura del manuscrito, su interés y sus valiosos comentarios. Asimismo, Florencia Gordón nos ayudó con la búsqueda bibliográfica, Solana García Guraieb con la traducción del resumen y Pedro Tiberi con la confección del mapa. Queremos agradecer también al Sr. Arturo Olivero, de la Ea. La Carlina, a la Flia. Nuevo Freyre (Ea. Las Tunas y La Carlina) y a la Flia. Hilden (Ea. Cerro Bayo) por las facilidades brindadas para la realización del trabajo y por su hospitalidad.

El trabajo fue realizado en el marco de los proyectos "Poblamiento humano y paleoambientes de las cuencas lacustres esteparias: arqueología de los lagos Cardiel y Strobel", (PICT 98 N $^{\circ} 4511$ ) y "Distribuciones, tecnología y poblamiento humano en la cuenca de los lagos Cardiel y Strobel (Santa Cruz)" (UNPA-UARG 29/A114). 


\section{BIBLIOGRAFÍA}

ALONSO AGUIRRE, A., C. BRÔSER y T. MÔRNER. 1999. Descriptive epidemiology of roe deer mortality in Sweden. J ournal of Wildlife Diseases 35 (4): 753762.

BARTON, C. M y G. A. CLARK. 1993. Cultural and Natural Formation Processes in Late Quaternary Cave and Rockshelter sites of western Europe and the Near East. En Formation Processes in Archaeological Context. Editado por P. Goldberg, D. T. Nash y M. D. Petraglia. Monographs in World Archaeology 17: 33-52. Prehistory Press, Madison, Wisconsin.

BEHRENSMEYER, A. K. 1975. Taphonomy and paleoecology in the hominid fossil record. Yearbook of Physical Anthropology 19: 36-50.

BEHRENSMEYER, A. K. 1978. Taphonomic and Ecologic Information from Bone Weathering. Paleobiology 4: 150-162.

BEHRENSMEYER, A. K. 1983. Patterns of natural bone distribution on recent land surfaces: implications for archaeological site formation. En Animals and Archaeology, Vol. 1. Hunters and Their Prey. Editado por J. Clutton-Brock y C. Grigson. Oxford: BAR International Series 163: 93-106.

BEHRENSMEYER, A. K. y D. DECHANT-BOAZ. 1980. The recent bones of Amboseli National Park, Kenya, in relation to East African Paleoecology. En Fossils in the Making. Editado por A. K. Behrensmeyer y A. P. Hill, pp 72-92. Chicago, IL: University of Chicago Press.

BEHRENSMEYER, A. K., K. D. GORDON y G. T. YANAGI. 1986. Trampling as a Cause of Bone Surface Damage and Pseudo-Cutmarks. Nature 319: 768771.

BERGER, J . 1983. Ecology and catastrophic mortality in wild horses: implications for interpreting fossil assemblages. Science 220: 1403-1404.

BINFORD, L. R. 1978. Nunamiut Ethnoarchaeology. Academic Press, New York.

BINFORD, L. R. 1981. Bones: Ancient Men and Modern Myths. Academic Press, New York.

BINFORD, L. R. 1989. En busca del pasado. Editorial Crítica, Barcelona.

BONINO, N. 1988. Censo de las poblaciones de guanacos en Tierra del Fuego; datos preliminares. En Primeras J ornadas Nacionales de Fauna Silvestre. La Pampa, Argentina.

BORRERO, L. A. 1988a. Estudios tafonómicos en Tierra del Fuego: su relevancia para entender procesos de formación del registro arqueológico. En Arqueología Contemporánea Argentina. Actualidad y Perspectivas. Editado por H. Yacobaccio, pp. 13-32. Editorial Búsqueda, Buenos Aires.

BORRERO, L. A. 1988b. Tafonomía Regional. En De Procesos, Contextos y otros Huesos. Editado por N. R. Ratto y A. F. Haber, pp. 9-15. Instituto de Ciencias Antropológicas, Universidad de Buenos Aires.

BORRERO, L. A. 1989. Sites in action: the meaning of guanaco bones in Fuegian Archaeological sites. Archaeozoologia 3: 9-24.

BORRERO, L. A. 1990. Fuego-Patagonian Bone Assemblages and the Problem of Communal Guanaco Hunting. En H unters of the Recent Past, editado por L. B. Davis y B. O. K. Reeves, pp. 373-399. Unwyn Hyman, London.

BORRERO, L. A. 2001. Regional Taphonomy: Background noise and the Integrity of the Archaeological Record. En Ethnoarchaeology of Andean South America. Contributions to archaeological Method and Theory, Editado por L. A. Kuznar, pp 243-254. International Monographs in Prehistory. Ethnoarchaeological Series 4.

BORRERO, L. A., F. M. MARTIN y J. VARGAS. 2005. Tafonomía de la interacción entre pumas y guanacos en el Parque Nacional Torres del Paine, Chile. Magallania 33 (1): 95-114.

BRAIN, C. K. 1981. The hunters or the hunted?. An Introduction to African Cave Taphonomy. Chicago, University of Chicago Press.

CAJAL, J. L. y R. A. OJ EDA. 1994. Camélidos Silvestres y Mortalidad por tormentas de nieve en la cordillera frontal de la Provincia de San Juan, Argentina. Mastozoología Neotropical 1(1): 81-88.

CAPALDO, S. D. y C. R. PETERS. 1995. Skeletal Inventories from Wildebeest Drownings at Lakes Masek and Ndutu in the Serengueti Ecosystem of Tanzania. J ournal of Archaeological Science 22: 385-408.

CASAMIQUELA, R. 1983. La significación del guanaco en el ámbito pampeano-patagónico; aspectos cronológicos, ecológicos, etológicos y etnográficos. Mundo Ameghiniano 4: 20-46.

CHILDS, H. 1997. El J immy. Fugitivo de la Patagonia. Editorial Zaguier \& Urrutia publications. Ushuaia, Argentina.

CONYBEARE, A. y G. HAYNES. 1984. Observations on elephant mortality and bones in water holes. Quaternary Research 22: 189-200.

CLUBB, R. y D. MACDONALD. 2004. The effects of capture, handling and husbandry in the welfare of South American camelids in Chile. MACS Project, Work Package 1, Final Report. htpp: //www.macs.puc.c/ publications/macswildcrureport.pdf. 19/08/2005.

CRUZ, I. 1999. Estepa y bosque: paisajes actuales y tafonomía en el noroeste de Santa Cruz. La utilización de lagos y lagunas en la región de lago Argentino (Provincia de Santa Cruz). En Soplando en el viento. Actas de las III J ornadas de Arqueología de la Patagonia. Pp. 303-314. Universidad Nacional del Comahue.

DARWIN, C. 1951. Viaje de un naturalista alrededor del mundo. Editorial El Ateneo, Buenos Aires, Argentina.

De LAMO, D. A. 1995. Aspectos ecofisiológicos. En Técnicas para el manejo del Guanaco. Editado por S. Puig. Cap. 6: 85-95. UICN. Gland, Suiza.

De LAMO, D. A., J. GARRIDO y J. ARES. 1982. Estructura de edades de la población de guanacos (Lama guanicoe Muller, 1776, Mammalia Camelidae). En 10² Reunión Argentina de Ecología, Mar el Plata, Argentina.

DUMBAR, M., R. VELARDE, M. A. GREG G y M. BRAY. 1999. Health evaluation of a pronghorn antelope population in Oregon. J ournal of Wildlife Diseases 35 (3): 495510.

DYNESIUS, M. y R. JANSSON. 2000. Evolutionary consequences of changes in species geographical distributions driven by Milankovitch Climate Oscillations. Proceedings of the National Academy of Sciences 97 (16): 9115-9120. 
ENDLICHER, W. y A. SANTANA AGUILA. 1988. El clima del sur de la Patagonia y sus aspectos ecológicos. Un siglo de mediciones climatológicas en Punta Arenas. Anales del Instituto de la Patagonia, Serie Ciencias Naturales 18: 57-86.

ENDLICHER, W. y A. SANTANA AGUILA. 1997. El invierno de 1995: un fenómeno climático muy severo en la Patagonia austral. Anales del Instituto de la Patagonia, Serie Ciencias Naturales 25: 77-88.

FIORILLO, A. R. 1989. An experimental study of trampling: implications for the fossil record. En Bone Modification. Editado por R. Bonnichsen y M. H. Sorg, pp 61-71. Orono: Center for the Study of the First Americans.

FITZ ROY, R. 1839. Narrative of the Surveying Voyages of $\mathrm{H}$ is Majesty's Ships "Adventure" and "Beagle", Between the years 1826 and 1836, describing their examination of the southern shores of South America and the "Beagle" Circumnavigation of the globe. Vol. I y II y apéndice. Londres.

FRANKLIN, W.L. 1982. Biology, ecology, and relationship to man of the South American camelids. En: Mammalian Biology in South America. Editado. por H. Mares y M. G. Genoways. University of Pittsburgh. Special Publication Series, Volume 6, pp. 457-488. Pymatuning. Laboratory of Ecology.

FRANKLIN, W.L. 1983. Contrasting socioecologies of South American's wild camelids: the vicuña and guanaco. En Advances in the study of mammalian behaviour Editado por J . F. Eisenberg y D.. Kleiman, American Society of Mammologists. Special Publication $\mathrm{N}^{\circ} 7$ : 573-629.

GONZÁLEZ, F. 1965. Diario del viaje que se hizo por Tierra del Puerto Deseado al Río Negro. 1798. Acad. Nac. Hist., Biblioteca XXIII.

GADER, R., \& DEL VALLE, A. 1982. Relevamiento aéreo de guanacos en el Departamento de Collón-Curá. Neuquén, Argentina. Dirección General de Recursos Faunísticos. MS.

GOÑI, R. A. 1988. Arqueología de momentos tardíos en el Parque Nacional Perito Moreno (Santa Cruz, Argentina). Precirculados de las ponencias científicas presentada a los simposios. IX Congreso Nacional de Arqueología Argentina, pp. 140-151. Facultad de Filosofía y Letras, UBA.

GOÑI, R., S., ESPINOSA, J . B. BELARDI, R., MOLINARI, F. SAVANTI, A. ARAGONE, G. CASSIADORO, G. LUBLIN y D. RINDEL. 2000. Poblamiento de la estepa patagónica: cuenca de los lagos Cardiel y Strobel. Actas del XIII Congreso Nacional de Arqueología Argentina. Córdoba. En prensa.

GUTIERREZ, M. y C. A. KAUFMANN. 2004. El rol de la acción fluvial en los procesos de formación del sitio Paso Otero 1. Resúmenes del XV Congreso Nacional de Arqueología Argentina, pp 179-180. Río Cuarto, Córdoba.

HALSTEAD, P. y J. O'SHEA. 1989. Introduction: Cultural Responses to risk and uncertainty. En Bad year economics: Cultural Responses to Risk and Uncertainty. Editado por P. Halstead y J. O'Shea. Capítulo 1: 1-7. Cambridge University Press, Cambridge.

HATCHER, J. B. 2003 [1903]. Cazadores de huesos en la Patagonia. Expediciones de la universidad de Princeton a la Patagonia. Marzo 1896 a Setiembre 1899. Zagier y Urruty, Ushuaia, Argentina.
HAYNES, G. 1982. Utilization and Skeletal Disturbances of North American Prey Carcasses. Artic 35 (2): 266281.

HAYNES, G. 1988. Mass deaths and serial predation: comparative taphonomic studies of modern large mammal death sites. Journal of Archaeological Science 15: 219-235.

HAYNES, G. 1995. Pre-Clovis and Clovis Megamammals: a comparison of carcass disturbance, age profiles and other characteristics in light of recent actualistic studies. En Ancient Peoples and Landscapes. Editado por E. J onson, pp. 9-27. Museum of Texas, Tech University, Lubbock.

HILL, A. P. 1980. Early postmortem damage to the remains of some contemporary East African mammals. En Fossils in the making: Vertebrate Taphonomy and Paleoecology. Editado por A. K. Behrensmeyer y A. P. Hill, pp. 131-152. Chicago: University of Chicago Press.

HUDSON, W. H. 1953. Un naturalista en el Plata. El Elefante Blanco, Buenos Aires.

KAUFMANN, C. A. 2004. La fusión ósea como indicador de edad y estacionalidad en guanaco (Lama guanicoe). En Contra viento y marea. Arqueología de la Patagonia. Editado por M. T. Civalero, P. M. Fernández y A. G. Guráieb, pp. 477-487. Instituto Nacional de Antropología y Pensamiento Latinoamericano y Sociedad Argentina de Antropología. Buenos Aires.

KLEIN, D. R. 1968. The introduction, increase and crash of reindeer on St. Matthew Island. J ournal of Wildlife Management 32: 350-367.

LARRIEU, E., BAGATTI, R y OPORTO, N. 1983. Mortalidad en guanacos juveniles criados en cautividad. Revista Therios. 1(1): 42-44. Argentina.

L'HEREUX, G. L. y L. A. BORRERO. 2002. Pautas para el reconocimiento de conjuntos óseos antrópicos y no antrópicos de guanaco en Patagonia. Intersecciones en Antropología 3: 29-40.

LUBINSKI, P. M. y C. J . O 'BRIEN. 2001. Observations on seasonality and mortality from a recent catastrophic death assemblage. J ournal of Archaeological Science 28: 833-842.

MADSEN, A. 1998. La Patagonia Vieja. Editorial Zaguier \& Urruty. Ushuaia, Argentina.

MARTIN, F. M. y L. A. BORRERO. 1997. A Puma lair in Southern Patagonia: implications for the archeological record. Current Anthropology 38(3): 453461.

MARTINKA, C. 1967. Mortality of northern Montana pronghorns in a severe winter. J ournal of Wildlife Management 31: 159-164.

MELTZER, D. J . 2003. Lessons in Landscape Learning. En Colonization of Unfamiliar Landscapes: The archaeology of adaptation. Editado por M. Rockman y J. Steele, pp. 222-241. Routledge, London y New York.

MENDOZA, D. 1965. Diario y memoria del viaje al Estrecho de Magallanes 1865-1866. Buenos Aires. Con prólogo y notas de M. A. Vignati. Acad. Nac. H ist, Biblioteca XXIV.

MENGONI GOÑLONS, G. 1995. La importancia socioeconómica del guanaco en el período precolombino. En Técnicas para el manejo del Guanaco. Editado por S. Puig. Capítulo 1: 13-25. UICN. Gland, Suiza. 
MERINO, M. L. 1988. Estructura social de la población de guanacos en la costa norte de Península Mitre, Tierra del Fuego, Argentina. Cuartas J ornadas Argentinas de Mastozoología. Tucumán, Argentina.

MERIN O, M. L y J. L. CAJAL. 1993. Estructura Social de la Población de Guanacos (Lama guanicoe Muller, 1776) en la costa norte de Península Mitre, Tierra del Fuego, Argentina. Studies on Neotropical Fauna and Environment 28: 129-138.

MICOZZI, M. S. 1986. Experimental study of postmortem change under field conditions: effects of freezing, thawing and mechanical injury. J ournal of Forensic Sciences 31 (3): 953-961.

MICOZZI, M. S. 1991. Postmortem Change in Human and Animal Remains. A Sistematic Approach. Charles C. Thomas Publisher, Springfield, Illinois, USA.

MINC, L. D. y K. P. SMITH. 1989. The Spirit of survival: cultural responses to resource variability in North Alaska. En Bad year economics: Cultural Responses to Risk and Uncertainty. Editado por P. Halstead y J . O'Shea. Capítulo 2: 8-39. Cambridge University Press, Cambridge.

MONDINI, N. M. 1994. Artiodactyl prey transport by foxes in puna rock shelters. Current Anthropology 36 (3): 520-524.

MONTES, C., D. A. De LAMO y J. ZAVATTI. 2000. Distribución de abundancias de guanaco (Lama guanicoe) en los distintos ambientes de Tierra del Fuego, Argentina. Mastozoología Neotropical 7 (1): 5-14.

MORENO, F. P. 1969. Viaje a la Patagonia austral 1876-1877. Solar-H achette, Buenos Aires.

MUSTERS, G. CH. 1997. Vida entre los patagones. El Elefante Blanco, Buenos Aires.

NASTI, A. 1994-95. Desarticulación natural y supervivencia de partes anatómicas: Tafonomía de vertebrados modernos en medioambientes puneños. Palimpsesto. Revista de Arqueología 4: 70-89.

O 'CONNELL, J. F., HAWKES, K. y N. G. BLURTON J ONES. 1988. Hadza hunting, butchering and bone transport and their archaeological implications. Journal of Anthropological Research 44: 113-161.

O 'HARA, T. M., G. CARROLL, P. BARBOZA, K. MUELLER, J. BLAKE, V. WOSHNER y C. WILETTO. 2001. Mineral and heavy metal status as related to a mortality event and poor recruitment in a moose population in Alaska. J ournal of Wildlife Diseases 37 (3): 495-510.

OPORTO, N. R. 1983. Contribución al conocimiento sobre el comportamiento de guanaco y posibles aplicaciones. Argentina. Mundo Ameghiniano 4: 1-19.

ORTEGA, I. 1985. Social organization and ecology of migratory guanaco population in Southern Patagonia. Ames, lowa, USA lowa State University. Master thesis.

PATERSON, S., K. WILSON y J. M. PEMBERTON. 1998. Major histocompatibility complex variation associated with juvenil survival and parasite resistance in a large unmanaged ungulate population (Ovis aries L.). Procedures of the National Academy of Sciences 95: 3714-3719.

PICKERING, T. R. 2002. Reconsideration of criteria for differentiating faunal assemblages accumulated for hyenas and hominids. International J ournal of Osteoarchaeology 12: 127-141.
PISANO, E. V. 1989-90. Labilidad de los ecosistemas terrestres Fuego-Patagónicos. Anales del Instituto de la Patagonia, Serie Ciencias Naturales 19: 17-25.

PRICHARD, H. H. 2003 [1903]. En el Corazón de la Patagonia. Editorial Zagier y Urruty. Ushuaia, Argentina.

PUIG, S. 1986. Ecología poblacional del guanaco (Lama guanicoe, Camelidae, Artiodactyla) en la Reserva Provincia de La Payunia (Mendoza). Argentina. Tesis doctoral, Universidad de Buenos Aires.

PUIG, S. y F. VIDELA. 1995. Comportamiento y organización social del guanaco. En Técnicas para el manejo del Guanaco. Editado por S. Puig. Cap. 7: 97-118. UICN. Gland, Suiza.

RAEDEKE, K. J. 1976. El guanaco de Magallanes, Chile. Distribución y Biología. Corporación Nacional Forestal de Chile. Ministerio de Agricultura. Publicación Técnica $\mathrm{N}^{\circ} 4$.

RAMOS, V. A. 1982. Geología de la región del Lago Cardiel, Provincia de Santa Cruz. Revista Asociación Geológica Argentina, XXXVII (1): 33- 49.

REIMERS, E. 1982. Winter mortality and population trends of reindeer on Svalbard, Norway. Artic and Alpine Research 14: 295-300.

ROCKMAN, M. y J. STEELE (Editores). 2003. Colonization of Unfamiliar Landscapes: The archaeology of adaptation. Routledge, London y New York.

ROWLEY-CONWY, P. y M. ZVELEBIL. 1989. Saving it for later: storage by prehistoric hunter-gatherers in Europe. En Bad year economics: Cultural Responses to Risk and Uncertainty. Editado por P. Halstead y J . O'Shea. Capítulo 3: 40-56. Cambridge University Press, Cambridge.

De RUITER, D. J y L. R. BERGER. 2001. Leopard (Panthera pardus Linneaus) cave caching related to anti-theft behavior in the John Nash Nature Reserve, South Africa. African J ournal of Ecology 39: 396-398.

SABA, S. 1987. Biología reproductiva del guanaco (Lama guanicoe Müller). Tesis Doctoral. Universidad Nacional de La Plata. La Plata. Argentina.

SABA, S., De LAMO, D. y S. PUIG. 1995. Dinámica poblacional del guanaco. En Técnicas para el manejo del Guanaco. Editado por S. Puig. Capítulo 5: 7183. UICN. Gland, Suiza.

SARNO, R. J., W. R. CLARK, M. S. BANK, W. S. PREXL, M. J. BEHL, W. E. J OHNSON y W. L. FRANKLIN. 1999. Juvenile guanaco survival: management and conservation implications. Journal of Applied Ecology 36: 937-945.

SAVANTI, F., T. BOURLOT y A. ARAGONE. 2005 Zooarqueología y uso del espacio en lago Cardiel, Provincia de Santa Cruz, Patagonia argentina. Archaeofauna 14: 111-127.

SAX, D. F. 2001. Latitudinal gradients and geographic ranges of exotic species: implications for Biogeography. J ournal of Biogeography 28: 139-150.

SCHALLER, G. y R. JUNRANG. 1988. Effects of a snowstorm on tibetan antelope. Journal of Mammalogy 69 (3): 631-634.

SHIPMAN, P. 1975. Implications of drought from vertebrate fossil assemblages. Nature 257: 667-668.

STRAUS, L. G. 1990. Underground Archeology: Perspectives on Caves and Rockshelters. Theory and Method in Archaeology. Editado por M. B. Schiffer, Volumen 2: 255-304. University of Arizona Press, Tucson. 
STURZENBAUM, P. y P. BORRELI. 2001. Manejo de riesgos climáticos. En Ganadería Ovina Sustentable en la Patagonia Austral. Tecnologías de Manejo Extensivo. Editado por P. Borreli y G. Oliva. Capítulo 13: 255-270. INTA- Santa cruz. Editorial ErreG é \& Asociados, Buenos Aires.

TILMAN, D., C. L. LEH MAN y K. T. THOMSON. 1997. Plant diversity and ecosystem productivity: Theoretical considerations. Proceedings of the National Academy of Science 94: 1857-1861.

TOOTS, H. 1965. Sequence of disarticulation in mammalian skeletons. University of Wyoming Contributions in Geology 4 (1): 37-39.

VILLALBA, R. 1994. Tree Ring and the Glacial Evidence from the Medieval Warm Epoch and the Little Ice Age in Southern South America. Climatic Change 26: 183-197.

VOORHIES, M. R. 1969. Taphonomy and population dynamics of an early Pliocene fauna, Knox County, Nebraska.
Contributions to Geology, Special Paper No. 1. Laramie, WY: University of Wyoming, pp. 1-69.

WAIDE, R. B., M. R. WILLING, C. F. STEINER, G. MITTELBACH, L. GOUGH, S. I. DODSON, G. P. JUDAY y R. PARMENTER. 1999. The Relationship between Productivity and Species Richness. Annual Review of Ecology and Sistematics 30: 257-300.

WEIGELT, J. 1989. Recent Vertebrate carcass and their archaeological implications. Chicago, IL: University of Chicago Press.

YELLEN, J. E. 1977 a. Archaeological approaches to the present: models for reconstructing the past. New York: Academic Press.

YELLEN, J . E. 1977 b. Cultural patterning in faunal remains: evidence from the !Kung Bushmen. En Experimental archaeology. Editado por D. W. Ingersoll y J. E. Yellen, pp.: 271-331. New York: Columbia University Press. 